\title{
Two-Dimensional Optical Orthogonal Codes for Fiber-Optic CDMA Networks
}

\author{
E. S. Shivaleela, A. Selvarajan, Member, IEEE, and T. Srinivas, Member, IEEE
}

\begin{abstract}
Several constructions of two-dimensional (2-D) codes have been proposed to overcome the drawbacks of nonlinear effects in large spread sequences of one-dimensional (1-D) unipolar codes in fiber-optic code-division multiple-access (FO-CDMA) networks. Wavelength-time (W/T) encoding of the 2-D codes is practical in FO-CDMA networks. W/T codes reported so far can be classified mainly into two types: 1) hybrid codes, where one type of sequence is crossed with another to improve the cardinality and correlation properties and 2) conversion of 1-D sequences to 2-D codes to reduce the "timelike" property. This paper describes the basic principles of a new family of wavelength/time multiple-pulses-per-row (W/T MPR) codes, for incoherent FO-CDMA networks, which have good cardinality, spectral efficiency, and minimal cross correlation values. In addition, an expression for the upper bound on the cardinality of W/T MPR codes is derived. Another feature of the W/T MPR codes is that the aspect ratio can be varied by a tradeoff between wavelength and temporal lengths. The correlation properties of W/T MPR codes are verified by simulation using Matlab. For given wavelength $\times$ time dimensions, various W/T codes, whose cardinalities are known, are compared, and it is shown that the W/T MPR family of codes have better cardinality and spectral efficiency than the other W/T codes. Performance analysis of the W/T MPR codes and their limiting cases is carried out for various parameter variations such as the dimensions of wavelength, time, and weight of the code.
\end{abstract}

Index Terms-Fiber-optic communication, incoherent optical communications, optical-code-division multiple access, wavelength/time codes.

\section{INTRODUCTION}

$\mathbf{P}$ ASSIVE broadcast star optical networks have been considered the best choice for high-speed access and local area networks (LANs). Optical-code-division multiple access (O-CDMA) is one such asynchronous multiplexing scheme with attractive features, such as concurrent access to the network, inherent security against interception, and the requirement of simple communication protocols. In incoherent fiber-optic code-division multiple-access (FO-CDMA) networks, unipolar $\{0,1\}$ codes are used. To keep the probability of error low due to multiple-access interference (MAI) in access networks, threshold (Th) at the receiver has to be set at a high value, and also codes that have low cross correlations are to be used. As the threshold value can be at most equal to the weight of the code $W$, codes with large weights $(1 s)$ are needed. Unipolar codes with low cross correlations can be obtained by

Manuscript received May 27, 2004; revised October 9, 2004.

The authors are with the Department of Electrical Communication Engineering, Indian Institute of Science, Bangalore-560 012, India (e-mail: lila@ece.iisc.ernet.in; rajan@ece.iisc.ernet.in; srinu@ece.iisc.ernet.in).

Digital Object Identifier 10.1109/JLT.2004.839974 minimizing the number of coincidences between 1s. In such codes, however, the length of the code increases rapidly when the number of users or the weight of the code is increased [1]. As the spread sequence length increases, ultrashort pulses have to be used, which are prone for nonlinear effects. To overcome this problem of one-dimensional (1-D) codes in O-CDMA, several types of encoding of the CDMA sequences, such as frequency-hopping $(\mathrm{FH})$, time-space $(\mathrm{T} / \mathrm{S})$, and wavelength-time (W/T), have been proposed. FH codes [2] are not suitable for FO-CDMA [3], as systems using the frequency-encoding CDMA technique suffer from optical beat noise that appears between the frequency slices at the photodetector [4] and [5]. Simulation results show that T/S systems are limited by the skew in associated ribbon fibers [6] and [7]. W/T coding of the CDMA sequence is suitable, and several W/T codes have been proposed, either by crossing one type of sequence with another, such as prime-hop [8], and extended quadratic congruence/prime (eqc/prime) [9] and [10] or converting 1-D sequences to two-dimensional (2-D) sequences [11]-[14]. In [11] and [13], Mendez et al. have described the design of pseudo-orthogonal codes based on folding of optimum Golomb rulers, and these codes have arbitrary cardinality. A technology demonstrator using these codes is described in [12]. Yang and Kwong [14] have constructed 2-D optical orthogonal signature pattern codes (OOSPC) from 1-D optical orthogonal codes (OOCs). The cardinality and correlation properties of OOSPCs is same as that of 1-D OOCs. Raymond et al. have described a depth-first search algorithm for the generation of W/T 2-D codes, which have out-of-phase autocorrelation $\lambda_{a}=1$ and peak cross correlation $\lambda_{c}=1$ [15]. In [16], Patel et al. have used wavelength-hopping prime codes, which have $\lambda_{a}=0$ and $\lambda_{c}=1$. In [17], we reported earlier the design of addition modulo $L_{T}$ (AML) codes, where $L_{T}$ is the temporal length. AML codes are single-pulse-per-row (SPR) codes with $\lambda_{a}=0$ and $\lambda_{c}=1$ values. As T/S systems are limited by the skew in associated ribbon fibers, AML codes can be encoded in W/T dimensions instead of T/S, as described in [17], where wavelength encoding is done in place of space. In this paper, AML codes encoded in W/T are hereafter called W/T SPR codes. This paper reports a new family of 2-D W/T multiple-pulses-per-row (W/T MPR) codes that have design flexibility in the choice of the weight/row $W_{p}$ for a given $W$ and, in addition, have 1) good cardinality, 2) good spectral efficiency, and 3) minimal correlation values for given wavelength and time dimensions.

Section II explains the basic principles for the construction of W/T MPR codes, to have minimal correlation values. For every code of the family of W/T MPR codes, the generation of two matrices $H$ and $V$ and the necessary conditions to be satisfied 
in order for the codes to have $\lambda_{a}=\lambda_{c}=1$ are given. An expression for the upper bound on the cardinality of W/T MPR codes is derived. It is also proven that a W/T MPR code of $R$ rows and weight/row $W_{p}$ can be constructed from a family of $R$ distinct OOCs of weight $W_{p}$ as row vectors. In Section III, the autocorrelation and cross-correlation properties of W/T MPR codes are verified by simulation using Matlab. Various W/T codes (whose cardinalities are known) are discussed and compared for cardinality, spectral efficiency, and $\lambda_{c}$ in Section IV. Section V compares the performances of W/T MPR codes with W/T SPR codes and OOCs, which are the limiting cases when $R=W$ and $R=1$, respectively. Performance analysis is carried out considering only MAI, as it is the major source of noise in FO-CDMA broadcast networks.

\section{BASIC PRINCIPLES OF W/T MPR CODES}

In this section, we describe the basic principles of the newly proposed W/T MPR codes, which are characterized by $N(R \times$ $L_{T}, W, \lambda_{a}=1, \lambda_{c}=1$ ), where $N$ is the number of codes, $R$ is the number of rows, $L_{T}$ is the number of columns, $W$ is the weight of the code, $W_{p}=W / R$ is the weight per row, $\lambda_{a}$ is the peak out-of-phase autocorrelation, and $\lambda_{c}$ is the peak cross correlation. In W/T MPR codes, the rows are encoded by distinct wavelengths, and the columns are encoded in time. The number of $1 s W_{p}$ in each of the rows of a W/T MPR code are assumed to be equal. Autocorrelation and cross correlation of W/T MPR codes are defined as follows.

Autocorrelation of a W/T MPR code $x(t)$ is defined as

$$
Z_{x, x}(l)=\sum_{m=1}^{R}\left(\sum_{n=1}^{L_{T}} x_{m, n} x_{m,(n+l) \bmod L_{T}}\right)
$$

$Z_{x, x}(l)$ satisfies

$$
Z_{x, x}(l)\left\{\begin{array}{ll}
=W, & \text { if } l=0 \\
\leq \lambda_{a}, & \text { if } 1 \leq l \leq L_{T}-1
\end{array} .\right.
$$

Cross correlation between two W/T MPR codes $x(t)$ and $y(t)$ is defined as

$$
Z_{x, y}(l)=\sum_{m=1}^{R}\left(\sum_{n=1}^{L_{T}} x_{m, n} y_{m,(n+l) \bmod L_{T}}\right) .
$$

$Z_{x, y}(l)$ satisfies

$$
Z_{x, y}(l) \leq \lambda_{c}, \quad \text { if } 0 \leq l \leq L_{T}-1
$$

where $x(t)$ and $y(t)$ are two periodic W/T MPR codes.

A W/T MPR code is represented by the chip positions of $1 \mathrm{~s}$ in each row, in the order of rows from $1,2, \ldots, R$. In Example 1, a W/T MPR code and its representation are shown. For convenience, in the remainder of the paper, unless specified otherwise, we refer to the representation of the codes itself as "codes." Also in this paper, we refer to $W_{p}$ as the weight/row in $\mathrm{W} / \mathrm{T}$ MPR codes and as weight of the code in OOCs, unless otherwise specified.

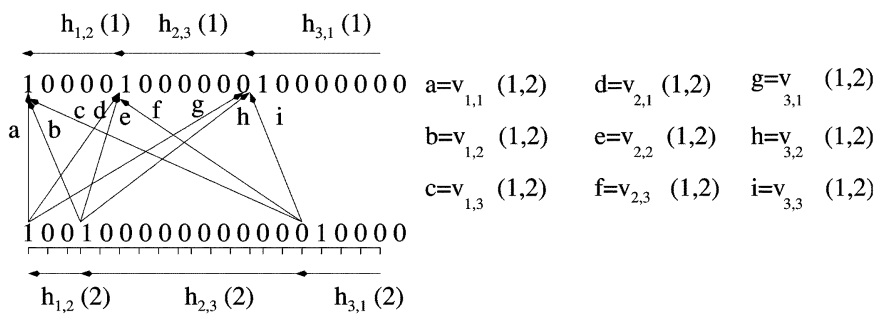

Fig. 1. Intra-row and inter-row distances between 1 s of a W/T MPR code.

Example 1: Representation of a W/T MPR code whose $L_{T}=10$ :

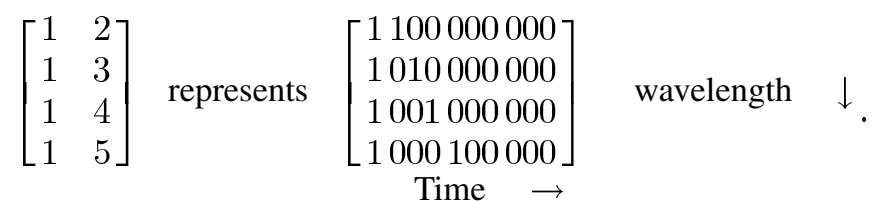

Example 2: W/T MPR codes for $R=4, W_{p}=3$, and $L_{T}=$ 64:

$$
\begin{gathered}
{\left[\begin{array}{lll}
1 & 2 & 13 \\
1 & 3 & 17 \\
1 & 4 & 21 \\
1 & 5 & 20
\end{array}\right]} \\
\text { code } 1
\end{gathered}
$$

The basic principles of the W/T MPR codes characterized by $\lambda_{a}=\lambda_{c}=1$ is explained hereafter. The following two definitions are useful in calculating the intra-row and inter-row distances between $1 \mathrm{~s}$ of a W/T MPR code. Fig. 1 illustrates the two types of distances between $1 \mathrm{~s}$ in a W/T MPR code.

Let $h_{k, l}^{a}(i)$ denote the distance between $l$ th and $k$ th pulses in $i$ th row of code $a$, as follows:

$$
h_{k, l}^{a}(i)= \begin{cases}l(i)-k(i), & \text { if }(i)>l k(i) \\ l(i)-k(i)+L_{T}, & \text { if } l(i)<k(i)\end{cases}
$$

where, $1 \leq l, k \leq W_{p}, l \neq k, 1 \leq i \leq R, 1 \leq l(i), k(i) \leq$ $L_{T}, l(i) \neq k(i), l(i)$, and $k(i)$ are the chip positions of the $l$ th and $k$ th pulses, respectively, in $i$ th row of code $a$.

Let $v_{k, l}^{a}(i, j)$ denote the distance between the $l$ th pulse of the $j$ th row and the $k$ th pulse of the $i$ th row of a W/T MPR code $a$, as follows:

$$
v_{k, l}^{a}(i, j)= \begin{cases}l(j)-k(i), & \text { if } l(j) \geq k(i) \\ l(j)-k(i)+L_{T}, & \text { if } l(j)<k(i)\end{cases}
$$

where $1 \leq l, k \leq W_{p}, 1 \leq i, j \leq R$, and $i \neq j, 1 \leq$ $l(j), k(i) \leq L_{T}, l(j)$, and $k(i)$ are the chip positions of the $l$ th and $k$ th pulses in the $j$ th and $i$ th rows, respectively, of code $a$.

Using the difference triangle property definitions (5) and (6), elements of $H$ and $V$ matrices are computed, and the two matrices are generated for each W/T MPR code of the family. Construction of $H^{a}$ and $V^{a}$ matrices for a W/T MPR code $a$ is explained with an example for a W/T MPR code of $R=3$ and $W_{p}=3$ in (7) and (8). The dimensions of $H$ and $V$ matrices are $R \times\left(W_{p}\right)\left(W_{p}-1\right)$ and $R(R-1) / 2 \times W_{p}^{2}$, respectively, as shown in (7) and (8) at the bottom of the next page. 


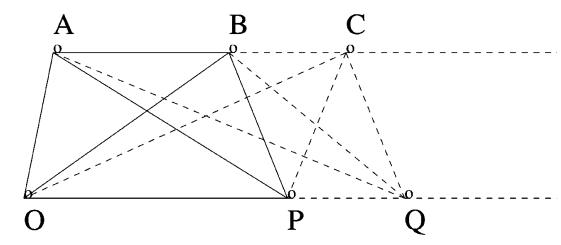

Fig. 2. Distances $h_{k, l}(i)$ and $v_{k, \hat{l}}(i, j)$ represented as the sides and $1 \mathrm{~s}$ as the vertices.

In order to satisfy the condition that $\lambda_{a}$ should be " 1 " in any W/T MPR code, the following conditions (1) and (2) are necessary.

(1) All the elements in the $i$ th row $1 \leq i \leq R$ of an $H$ matrix should be distinct.

(2) All the elements in the $i$ th row $1 \leq i \leq R(R-1) / 2$ of a $V$ matrix should be distinct.

For $\lambda_{c}$ to be "1" between any two W/T MPR codes, the following two conditions (3) and (4) are necessary.

(3) All the $i$ th row elements $1 \leq i \leq R$ of an $H$ matrix should be distinct from the $i$ th row elements of any other $H$ matrix.

(4) All the $i$ th row elements $1 \leq i \leq R(R-1) / 2$ of a $V$ matrix should be distinct from the $i$ th row elements of any other $V$ matrix.

In the limiting case, when $R=1$, a W/T MPR code is an OOC, since the previously described conditions reduce to (1) and (3) for $\lambda_{a}=1$ and $\lambda_{c}=1$, respectively. W/T MPR codes of $R$ rows, weight $W$, and weight/row $W_{p}=W / R$ can be constructed by using $R$ distinct OOCs of weight $W_{p}$, as row vectors. In the following Theorem 1 , by means of a geometrical proof, we prove that when two distinct OOCs of a family are chosen as row vectors of a W/T MPR code, condition (2) is satisfied.

Theorem 1: Between any pair of row vectors $R_{i}$ and $R_{j}, 1 \leq$ $i, j \leq R$, and $i \neq j$, of a W/T MPR code of $W_{p}=W / R, R_{i}$, and $R_{j}$ being distinct OOC words of weight $W_{p}$, all the $W_{p}^{2}$ $V_{k, \hat{l}}(i, j)$ distances are distinct (condition (2)).

Proof: Let $R_{i}$ and $R_{j}$ be any two distinct OOC words of the family of $N\left(L_{T}, W_{p}, \lambda_{a}=\lambda_{c}=1\right)$, and let them be the rows $i$ and $j$ of a W/T MPR code of $W_{p}=W / R$, where $1 \leq$ $i, j \leq R, i \neq j$ (see Fig. 2).

Let the $1 \mathrm{~s}$ in $R_{i}$ and $R_{j}$ be the vertices and the distances $h_{k, l}(i), h_{\hat{k}, \hat{l}}(j)$ and $v_{k, \hat{l}}(i, j)$ be the line segments between the vertices in Fig. 2. Let the vertices $A, B, C, \ldots$ represent the first, second, third, ... 1s, respectively, in $R_{i}$ and $O, P$, and $Q, \ldots$ represent first, second, third... 1s, respectively, in $R_{j}$. The vertices, in Fig. 2, $A, B, C, \ldots \epsilon G$ and $O, P, Q, \ldots \epsilon H$. Cardinality of $G=$ Cardinality of $H=W_{p}$. The distances $h_{k, l}(i)$ and $h_{\hat{k}, \hat{l}}(j)$ belong to the distinct rows $i$ and $j$, respectively, of the W/T MPR code, and hence we represent them by parallel lines. All the $h_{k, l}(i)$ and $h_{\hat{k}, \hat{l}}(j)$ distances in $R_{i}$ and $R_{j}$, respectively, are distinct due to the principle on which the OOCs are constructed [1].

Let $A B O P$ form a quadrilateral with $A B$ and $O P$ as the parallel sides and the other two sides $O A=V_{k, \hat{k}}(i, j)$ and $P B=V_{l, \hat{l}}(i, j)$.

Let us assume that $V_{k, \hat{k}}(i, j)=V_{l, \hat{l}}(i, j)$. Then, $h_{k, l}(i)=$ $h_{\hat{k}, \hat{l}}(j)$. However, this contradicts with the basic principles of OOCs [1]. Hence, $V_{k, \hat{k}}(i, j) \neq V_{l, \hat{l}}(i, j)$. Therefore, $O A$ and $P B$ sides are unequal and hence nonparallel. Therefore, $A B O P$ forms a trapezoid with $A B$ and $O P$ as the bases.

Next, let us assume the two diagonals $P A$ and $O B$ of the trapezoid $A B O P$ are equal. From Fig. 2, we have $P A=$ $V_{k, \hat{l}}(i, j)=\hat{l}(j)-k(i)$ and $O B=V_{l, \hat{k}}(i, j)=\hat{k}(j)-l(i)$; then $h_{\hat{k}, \hat{l}}(j)=h_{l, k}(i)$. This contradicts with the basic principles of OOCs. Hence, the two diagonals are unequal.

Next, $V_{k, \hat{l}}(i, j) \neq V_{k, \hat{k}}(i, j)$ since $\hat{k}(j) \neq \hat{l}(j) ; V_{l, \hat{k}}(i, j) \neq$ $V_{l, \hat{l}}(i, j)$ since $\hat{k}(j) \neq \hat{l}(j) ; V_{k, \hat{l}}(i, j) \neq V_{l, \hat{l}}(i, j)$ since $k(i) \neq$ $l(i) ; V_{l, \hat{k}}(i, j) \neq V_{k, \hat{k}}(i, j)$ since $k(i) \neq l(i)$. Hence, the two sides and the diagonals $O A, P B, P A$, and $O B$ are all distinct in $A B O P$.

By choosing two elements each from $G$ and $H$, we form $\left(\begin{array}{c}W_{p} \\ 2\end{array}\right) \times\left(\begin{array}{c}W_{p} \\ 2\end{array}\right)$ trapezoids in all. Keeping $V_{1,1}(i, j)$ as common, we prove that $W_{p}^{2} V_{k, \hat{l}}(i, j)$ distances are distinct from all the trapezoids.

Without loss of generality, let the elements $A$ and $O$ be chosen from the sets $G$ and $H$, respectively. Let $A$ and $O$ be the two vertices common to all the trapezoids and $V_{1,1}(i, j)$ be the distance between them. Let any other element, say $B$, be chosen from $W_{p}-1$ elements of $G$ (excluding $A$ ), as the third vertex. In the first step, we construct $W_{p}-1$ trapezoids, with $A, O$, and $B$ as the first, second, and third vertices, respectively, and for the fourth vertex, we choose an element from $W_{p}-1$ elements (excluding $O$ ) of the set $H$. Therefore, the number of distinct distances compared with $V_{1,1}(i, j)$ is equal to $1+2\left(W_{p}-1\right)$.

In the second step, we choose an element, say $C$ from $W_{p}-2$ elements of $G$ (excluding $A$ and $B$ ). We construct $W_{p}-1$ trapezoids with $A, O$, and $C$ as the first, second, and third vertices common to all. $W_{p}-1$ trapezoids are formed by choosing the elements from the set $H$ (excluding $O$ ), as the fourth vertex. Therefore, the number of distinct distances compared with $V_{1,1}(i, j)$ is equal to $1+\left(W_{p}-1\right)$. Similarly, other trapezoids are formed by choosing the third vertex from other $W_{p}-3$

$$
\begin{aligned}
H^{a}= & {\left[\begin{array}{llllllll}
h_{1,2}(1) & h_{2,3}(1) & h_{3,1}(1) & h_{1,3}(1) & h_{2,1}(1) & h_{3,2}(1) \\
h_{1,2}(2) & h_{2,3}(2) & h_{3,1}(2) & h_{1,3}(2) & h_{2,1}(2) & h_{3,2}(2) \\
h_{1,2}(3) & h_{2,3}(3) & h_{3,1}(3) & h_{1,3}(3) & h_{2,1}(3) & h_{3,2}(3)
\end{array}\right] } \\
V^{a}= & {\left[\begin{array}{lllllllll}
v_{1,1}(1,2) & v_{1,2}(1,2) & v_{1,3}(1,2) & v_{2,1}(1,2) & v_{2,2}(1,2) & v_{2,3}(1,2) & v_{3,1}(1,2) & v_{3,2}(1,2) & v_{3,3}(1,2) \\
v_{1,1}(1,3) & v_{1,2}(1,3) & v_{1,3}(1,3) & v_{2,1}(1,3) & v_{2,2}(1,3) & v_{2,3}(1,3) & v_{3,1}(1,3) & v_{3,2}(1,3) & v_{3,3}(1,3) \\
v_{1,1}(2,3) & v_{1,2}(2,3) & v_{1,3}(2,3) & v_{2,1}(2,3) & v_{2,2}(2,3) & v_{2,3}(2,3) & v_{3,1}(2,3) & v_{3,2}(2,3) & v_{3,3}(2,3)
\end{array}\right] . }
\end{aligned}
$$


elements of $G$ and the fourth vertex from $W_{p}-1$ elements of $H$. In all, in the second step, we form $\left(W_{p}-2\right)\left(W_{p}-1\right)$ trapezoids by choosing third and fourth vertices from $W_{p}-2$ elements of $G$ and $W_{p}-1$ elements of $H$, respectively, we compare $\left(W_{p}-2\right)+\left(W_{p}-2\right)\left(W_{p}-1\right)$ distinct $V_{k, \hat{l}}(i, j)$ distances with $V_{1,1}(i, j)$.

From steps 1 and 2 , the number of distinct distances compared with $V_{1,1}(i, j)$ is $1+2\left(W_{p}-1\right)+\left(W_{p}-2\right)+\left(W_{p}-2\right)\left(W_{p}-1\right)$, which is equal to $W_{p}^{2}-1$. Therefore, all the $W_{p}^{2}$ distances are distinct, which proves the theorem.

Corollary 1: A W/T MPR code of $R$ rows, $W$ weight, $\left(W_{p}=W / R\right)$ having $\lambda_{a}=1$, cannot have $R_{i}$ and $R_{j}$ as distinct row vectors, where $R_{i}$ is an OOC word of weight equal to $W / R$ and $R_{j}(k)=R_{i}\left((k+s) \bmod L_{T}\right)$, with a shift equal to $s, 0 \leq s \leq L_{T}-1$ and $1 \leq k \leq L_{T}$.

Corollary 2: A minimum number of OOCs required to construct a family of $N$ W/T MPR codes having $R$ rows is equal to $\lceil(N / R)\rceil R$ (assuming $N \geq R$ ).

Proof: From Theorem 1, we know that to construct a W/T MPR code of $R$ rows and weight $W$, the number of OOCs required is equal to $R$, of weight equal to $(W / R)=W_{p}$. A row vector $R_{i}$ of a W/T MPR code is a valid row vector $R_{j}, 1 \leq$ $i, j \leq R$ and $i \neq j$, of any other W/T MPR code in the family. Therefore, the minimum number of OOCs required to construct a family of $N \mathrm{~W} / \mathrm{T}$ MPR codes is equal to $\lceil(N / R)\rceil R$ (assuming $N \geq R$ ).

Next, we derive an expression for the lower bound on $L_{T}$ required to construct a family of $N \mathrm{~W} / \mathrm{T}$ MPR codes. First, we consider condition (1), condition (3), Theorem 1 and Corollary 2 and derive an expression for the temporal length $L_{T 1}$. Next, we consider conditions (2) and (4) and obtain another expression for the temporal length $L_{T 2}$. The maximum of $L_{T 1}$ and $L_{T 2}$ gives the required lower bound on the temporal length $L_{T}$.

For a W/T MPR code, we need $W_{p}\left(W_{p}-1\right)$ distinct distances for one row, due to condition (1). Since we have assumed $N \geq$ $R$, the number of distinct distances due to condition (3) is greater than or equal to that due to Theorem 1, i.e., $N \times W_{p}\left(W_{p}-\right.$ 1) $\geq R \times W_{p}\left(W_{p}-1\right)$. The number of distinct distances due to Corollary 2 is greater than or equal to that due to condition (3) i.e., $\lceil(N / R)\rceil \times R \times W_{p}\left(W_{p}-1\right) \geq N \times W_{p}\left(W_{p}-1\right)$. Hence, lower bound on $L_{T 1}$ is given by

$$
L_{T 1} \geq\left\lceil\frac{N}{R}\right\rceil \times R \times W_{p}\left(W_{p}-1\right)+1
$$

To satisfy condition (2), we need $W_{p}^{2}$ distinct distances between 1s of any pair of rows of a W/T MPR code, and to satisify condition (4), we need $N \times W_{p}^{2}$ distinct distances between $1 \mathrm{~s}$ of any pair of rows among the family of $N$ W/T MPR codes. It is interesting to note that $W_{p}^{2}$ distinct distances between a pair of rows need not all be distinct from $W_{p}^{2}$ distances of any other pair of rows of a W/T MPR code for $\lambda_{a}^{p}=1$.

Lower bound on $L_{T 2}$ is given by

$$
L_{T 2} \geq N \times W_{p}^{2}
$$

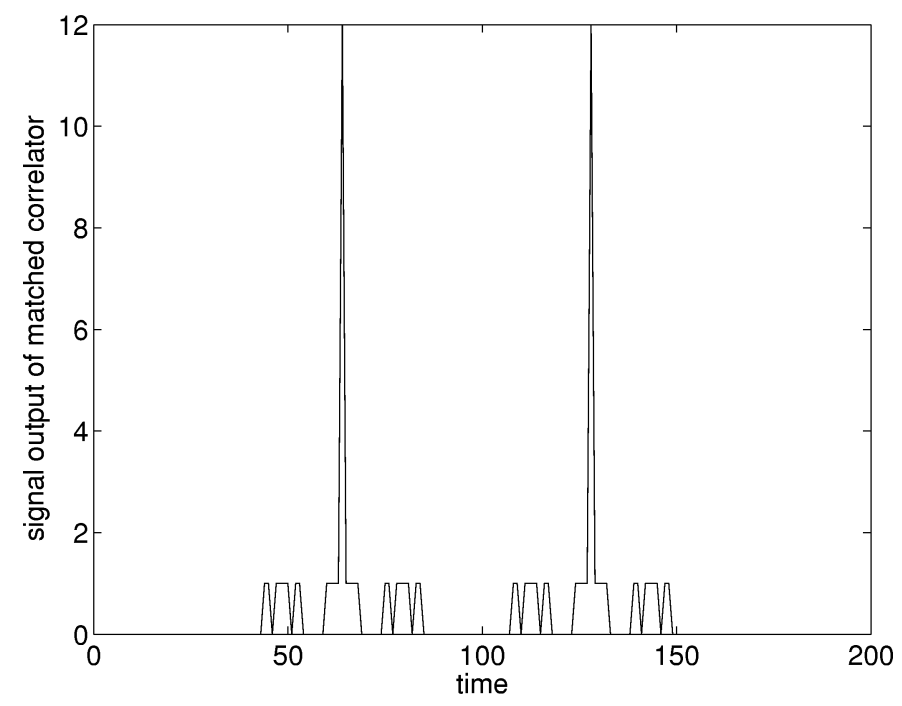

(a)

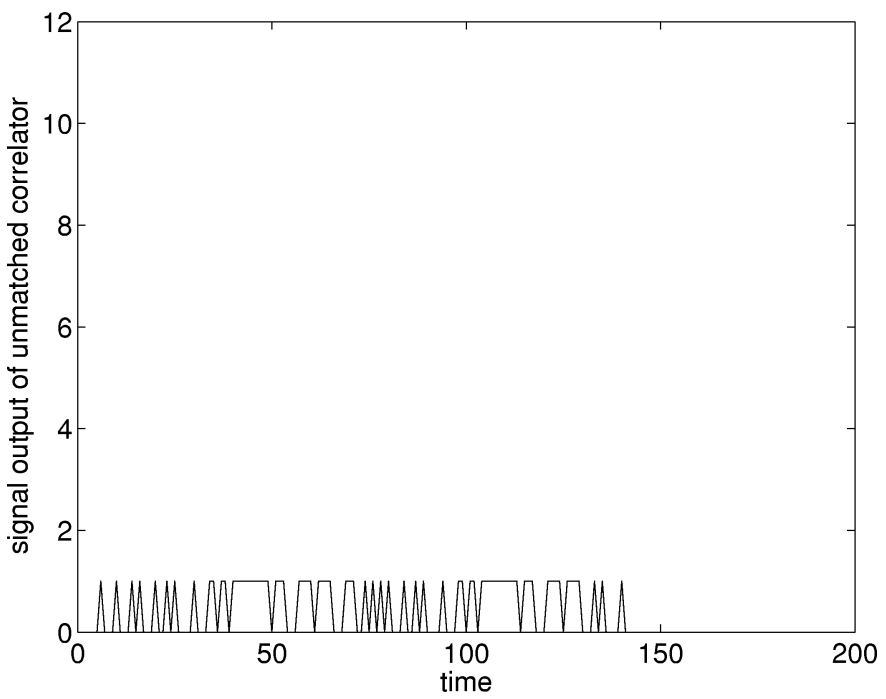

(b)

Fig. 3. (a) Autocorrelation and (b) Cross correlation of W/T MPR codes of Example 2 for bit pattern " 11 ".

From (9) and (10), lower bound on $L_{T}$ is given by

$$
L_{T} \geq \max \left\{L_{T 1}, L_{T 2}\right\} .
$$

The upper bound on the number of W/T MPR codes that can be constructed for a given $L_{T}$ is given by

$$
N \leq\left\lfloor\frac{L_{T}}{W_{p}^{2}}\right\rfloor
$$

In the next section, we verify the correlation properties of $\mathrm{W} / \mathrm{T}$ MPR codes.

\section{SimUlation}

We verify the autocorrelation and cross-correlation properties of W/T MPR codes by simulation using Matlab. The two W/T 
TABLE I

COMPARISON OF W/T CODES FOR CARDinAlity, SPECTRAL EFFICIENCY, AND PEAK CROSS CORRELATION

\begin{tabular}{l|r|r|r|r|r|r}
\hline Type of code & Weight & Row $\times$ Column & $\begin{array}{r}\text { Wave- } \\
\text { length }(\lambda)\end{array}$ & Cardinality & Spectral efficiency & $\lambda_{c}$ \\
\hline Prime-hop & $P_{s}=7$ & $1 \times P_{s}^{2}=1 \times 49$ & $P_{h}=7$ & $\left(P_{s}-1\right)\left(P_{h}-1\right)=36$ & $\frac{\left(P_{s}-1\right)\left(P_{h}-1\right)}{P_{h} \times P_{s}^{2}}=0.104$ \\
Eqc/prime & $P_{s}=5$ & $1 \times P_{s}\left(2 P_{s}-1\right)=$ & $P_{h}=7$ & $\left(P_{s}-1\right)\left(P_{h}-1\right)=24$ & $\frac{\left(P_{s}-1\right)\left(P_{h}-1\right)}{P_{h} \times P_{s}\left(2 P_{s}-1\right)}=0.076$ \\
\\
W/T SPR
\end{tabular}

MPR codes in Example 2 are chosen. Let $X(t)$ and $Y(t)$ be the two W/T MPR codes represented as matrices with $\{0,1\}$ entries and of dimensions $R(=4) \times L_{T}(=64)$. The elements in code 1 and code 2 in Example 2 determine the positions of $1 \mathrm{~s}$ in $X(t)$ and $Y(t)$, respectively. $X(t)$ and $Y(t)$ each have four wavelength channels, and the number of pulses or 1s per wavelength channel is equal to 3. Let $\hat{X}$ and $\hat{Y}$ represent the matched filters for the codes $X(t)$ and $Y(t)$, respectively. Matrices $\hat{X}$ and $\hat{Y}$ are generated by time inverting the " 1 " entries of $X(t)$ and $Y(t)$, respectively. For example, the first row vector of $\hat{X}$ has " 1 " entries in columns $L_{T}-1=63, L_{T}-2=62$ and $L_{T}-13=51$ and the remaining entries are " 0 "s. Let $X_{1}(t)=[X(t) X(t)]$ be the spread sequences for the bit sequence " 11 " using the code $X(t)$. We convolve the rows of matrix $X_{1}(t)$ with the corresponding rows of the matrix $\hat{X}$, according to (2), and add all the results of the convolved wavelength channels in time to get the autocorrelation for the code $X(t)$ for bit pattern " 11 ". Similarly, convolving the rows of the matrix $X_{1}(t)$ with the corresponding rows of the matrix $\hat{Y}$, according to (4), and adding all the results of convolved wavelength channels in time gives the cross correlation for bit pattern "11". The autocorrelation of code 1 and cross correlation between codes 1 and 2 in Example 2 are shown in the Fig. 3(a) and (b). We may note that in-phase autocorrelation is equal to the weight of the code $W=12$ and out-of-phase autocorrelation $\lambda_{a}=1$ in Fig. 3(a) and $\lambda_{c}=1$ in Fig. 3(b) for the two W/T MPR codes of Example 2.

In the next section, we compare W/T codes whose cardinalities are known, for cardinality, spectral efficiency, and $\lambda_{c}$.

\section{Discussion OF W/T CODES}

W/T codes proposed so far can be categorized mainly into two types: 1) hybrid sequences constructed by crossing one type of sequence with another, such as prime-hop [8] and eqc/prime [9] and [10] and 2) conversion of 1-D sequences to 2-D sequences [11]-[14]. We compare W/T codes, whose cardinalities are known, for cardinality, spectral efficiency, and $\lambda_{c}$ in Table I. For fair comparison, we choose the wavelength and time dimensions to be approximately the same for all the codes. The spectral efficiency of W/T codes is given by

$$
\text { Spectral efficiency }=\frac{N \times \frac{1}{T_{b}}}{R \times \frac{1}{T_{c}}}
$$

where $N$ is the cardinality, $T_{b}$ is the bit time, $T_{c}$ is chip time, and $R$ is the number of rows or wavelengths in a code. In [8], timespreading and wavelength-hopping hybrid prime-hop codes are described, where $\left(P_{s}-1\right)$ time-spreading prime sequences of length $P_{s}^{2}$ are crossed with $\left(P_{h}-1\right)$ wavelength-hopping prime sequences, where $P_{s}$ and $P_{h}$ are the prime numbers used in the algorithms for the generation of prime sequences, respectively. When $P_{s}=P_{h}$, the system is called "symmetric"; otherwise, it is asymmetric. Although symmetric prime-hop sequences have $\lambda_{c}=1$, for a general asymmetric system $\lambda_{c}>2$. Another type of hybrid codes, eqc/prime constructed in [9] and [10], have $\lambda_{c}=2$. In eqc/prime codes, $\left(P_{s}-1\right)$ time-spreading eqc sequences of length $P_{s}\left(2 P_{s}-1\right)$ are crossed with $\left(P_{h}-1\right)$ wavelength-hopping sequences. For fair comparison, we have computed the cardinalities of prime-hop and eqc/prime codes, under the same conditions, by crossing $\left(P_{s}-1\right)$ time-spread sequences with $\left(P_{h}-1\right)$ wavelength-hop sequences. Prime-hop codes have better spectral efficiency than eqc/prime codes. The spectral efficiency of the eqc/prime sequence is lower because of the increased sequence length $P_{s}\left(2 P_{s}-1\right)$ as compared with the $P_{s}^{2}$ length of prime-hop codes. When prime $P$ is used for the generation of W/T SPR codes, each code generated is a square matrix, but the number of rows considered in Table I is equal to the number of wavelengths $R=\lambda$. The cardinality of W/T SPR codes is equal to $P$, and the spectral efficiency is $(1 / R)$. Here, only rows $R_{0}, R_{1}, \ldots, R_{\lambda-1}$ are encoded with wavelengths $\lambda_{1}, \lambda_{2}, \ldots, \lambda_{\lambda}$, respectively. Although W/T MPR codes and OOSPCs are constructed from OOCs, both the codes differ in the method of construction. An OOC of weight $W$ is converted to an OOSPC of weight $W$ by using the Chinese remainder theorem, whereas a W/T MPR code of weight $W$ and $R$ rows is constructed from $R$ OOCs of weight $W_{p}$, chosen 
from a family of OOCs. The cardinality and correlation properties of OOSPCs is same as that of 1-D OOCs. In OOSPCs and W/T MPR codes, we have chosen $W=4, R=2$, and $L_{T}=49$ and 48 (approximately same), respectively. When the number of wavelengths $\lambda$ is greater than $R$, then the cardinality of W/T MPR codes and OOSPCs increases by $\lfloor(\lambda / R)\rfloor$, since only $R$ wavelengths are needed for one code. We have computed the cardinalities of W/T codes satisfying $\lambda_{c}$ listed against the respective codes in Table I. It can be seen from Table I that W/T MPR codes have the highest cardinality, spectral efficiency, and minimal correlation values for a given wavelength and time dimensions.

\section{Performance Analysis of FO-CDMA Networks USING DIFFERENT CODES}

In an asynchronous incoherent FO-CDMA using ON-OFF keying (OOK), with " 1 " or " 0 " sent with equal probability, only bit " 1 " is encoded by the CDMA sequence. We consider a simple protocol as in [1] to analyze the performance of FO-CDMA. An ideal link is considered in which the performance deterioration is only due to the MAI and the receiver noises, i.e., shot noise and thermal noise are ignored. The signal at the receiver contains the desired user's signal and the interference signal, $I$. $I$ is the sum of all the cross correlations that overlap in time at the receiver.

When any two W/T MPR codes of the same family having equal $W_{p}$ are correlated, there are $W_{p}^{2}$ possible overlaps in one row. Therefore, the total number of overlaps over all the rows $R$ is $R W_{p}^{2}$. Then, the probability of overlap for a W/T MPR code is $R W_{p}^{2} / L_{T}$. For a W/T MPR code, the probability of overlap in case of OOK is $\left(R W_{p}^{2}\right) /\left(2 L_{T}\right)$ and the complement of this event, i.e., the probability of no overlap, is $1-\left(R W_{p}^{2} / 2 L_{T}\right)$. In writing this, we have assumed that all the receivers are chip synchronized, i.e., either there is complete overlap of chips or there is no overlap. For W/T MPR codes, the probability density function $P_{I}$ for interference signal $I$ for the chip synchronous case is given by

$P_{I}=\sum_{i=0}^{N-1}\left(\begin{array}{c}N-1 \\ i\end{array}\right)\left(\frac{R W_{p}^{2}}{2 L_{T}}\right)^{i}\left(1-\frac{R W_{p}^{2}}{2 L_{T}}\right)^{N-1-i} \delta(I-i)$.

The upper bound on the probability of error/bit $P_{e}$ is evaluated by considering the chip-synchronous case in FO-CDMA networks.

\section{A. Probability of Error/Bit When Chips Are Synchronous}

The exact $P_{e}$ at a receiver is defined as

$$
\begin{aligned}
P_{e}(\text { exact })=P_{r}(I \geq & \operatorname{Th} / b=0) \cdot P_{r}(b=0) \\
& +P_{r}(I<\mathrm{Th} / b=1) \cdot P_{r}(b=1) .
\end{aligned}
$$

Because an FO-CDMA system with incoherent signal processing is a positive system, errors can occur only due to the

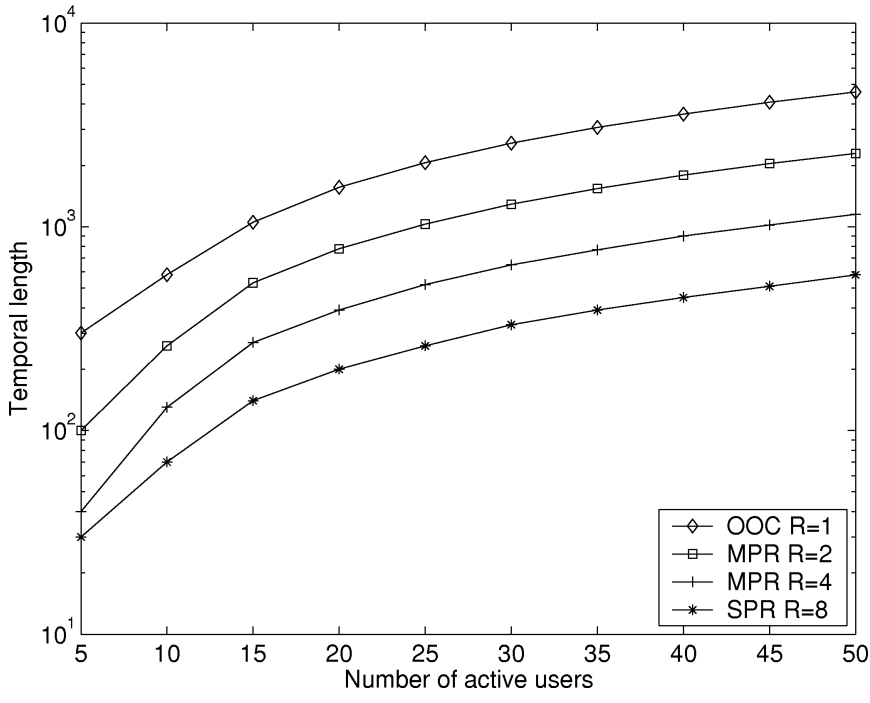

(a)

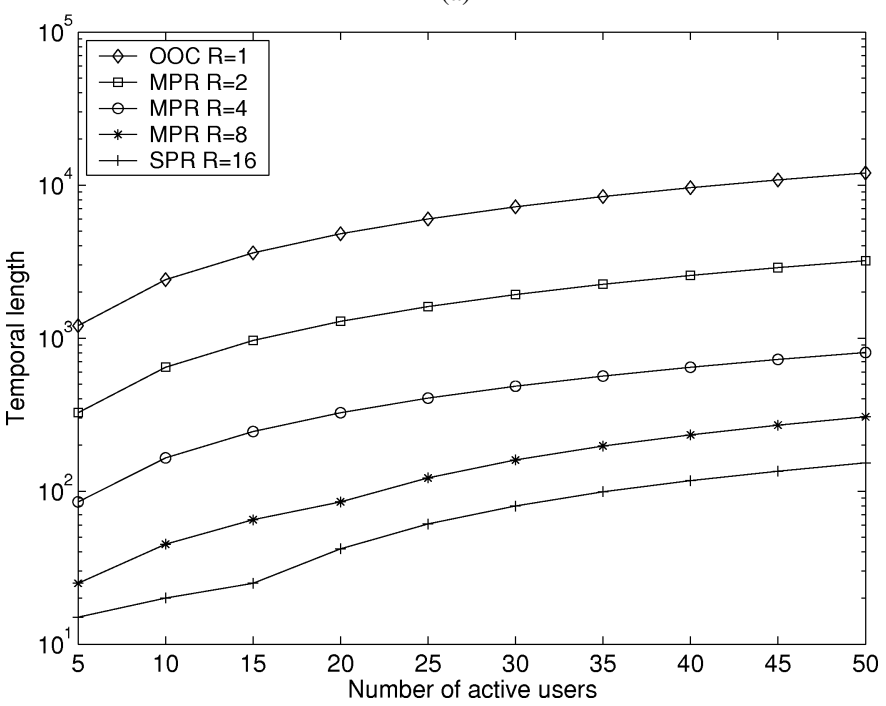

(b)

Fig. 4. Temporal lengths to achieve $P_{e}=10^{-9}$ when (a) $W=8$ and (b) $W=16$.

first term in (14). From (2), we know that the in-phase autocorrelation peak of 2-D codes and also of OOCs [1] are equal to $W$, and in all our analysis, we have considered $\mathrm{Th}=W$, as it is the optimum value to keep the errors due to MAI low [16].

$P_{e}$ for a W/T MPR code is given by

$$
P_{e}=\frac{1}{2} \sum_{i=\mathrm{Th}}^{N-1}\left(\begin{array}{c}
N-1 \\
i
\end{array}\right)\left(\frac{R W_{p}^{2}}{2 L_{T}}\right)^{i}\left(1-\frac{R W_{p}^{2}}{2 L_{T}}\right)^{N-1-i} .
$$

Temporal lengths needed for W/T MPR, W/T SPR codes, and OOCs is plotted in Fig. 4(a) and (b) for weights, $W=8$, and 16 respectively, using (15). We see that, for a given $W$, the $L_{T}$ needed to achieve a given $P_{e}$ decreases with the increase in the wavelength dimension for W/T MPR codes; for the limiting cases, when $R=W$, the $L_{T}$ is minimum, and when $R=$ 1, the $L_{T}$ is maximum. 


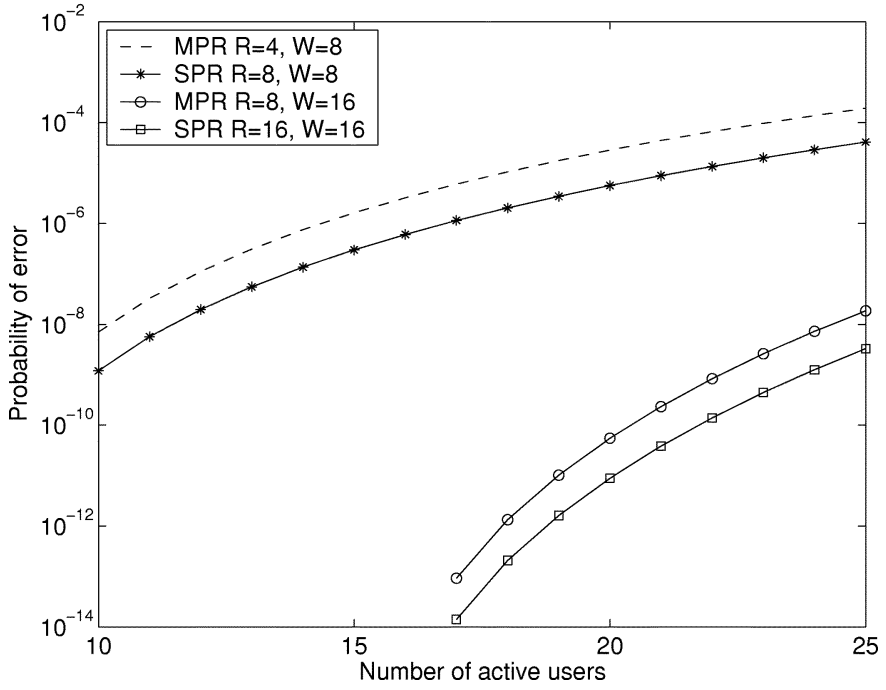

(a)

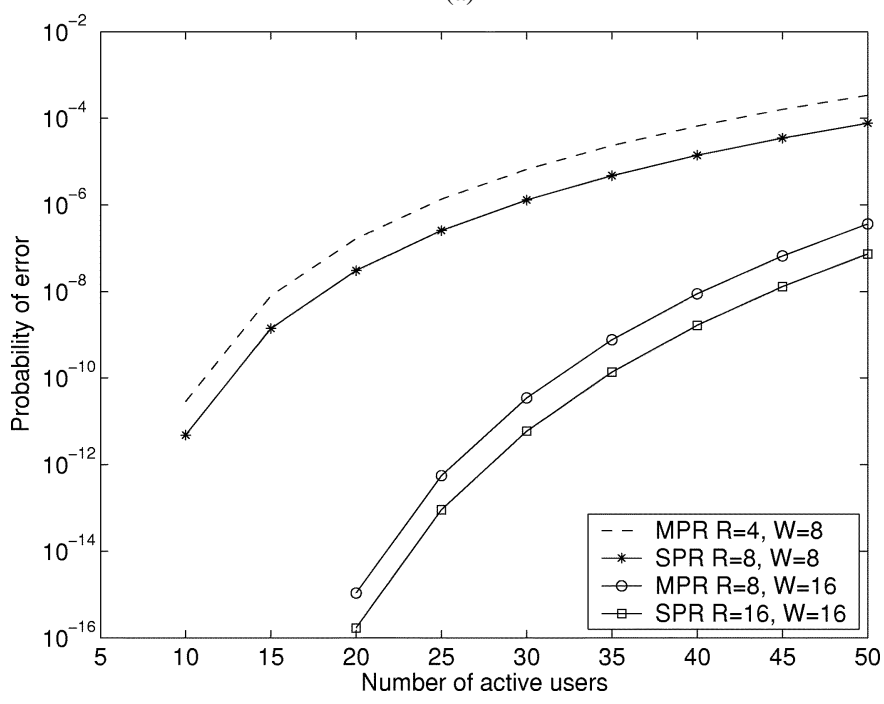

(b)

Fig. 5. Probability of error when $W_{p}=1$ and 2 , and $W=8$ and 16. (a) $L_{T}=100$. (b) $L_{T}=200$.

In Fig. 5(a) and (b), $P_{e}$ is plotted using (15) for $W=8$ and 16 and for $L_{T}=100$ and $L_{T}=200$, respectively, for W/T MPR and W/T SPR codes. Maximum values of $N$ are 25 and 50, for $L_{T}=100$ and 200, respectively, from (12) when $W_{p}=2$. $P_{e}=0$, in Fig. 5(a) and (b), when $\mathrm{Th}=W$ and $N<W$, since $I \leq N$, according to (15). It can be noted from these plots in Fig. 5(a) and (b) that, for a given $L_{T}, P_{e}$ decreases with the increase in $R$ and also $W$.

In Fig. 6(a) and (b), curves are drawn for constant $R=8$ and 16 , and $W$ is varied for W/T SPR and W/T MPR codes. It is observed from the plots in Fig. 6(a) and (b) that $L_{T}$ is minimum when $W=16$ for most part of the curves.

\section{CONCLUSION}

A new family of 2-D orthogonal W/T MPR codes have been proposed for FO-CDMA networks, and the basic principles of the codes to have minimal correlation values were explained. The autocorrelation and cross-correlation properties

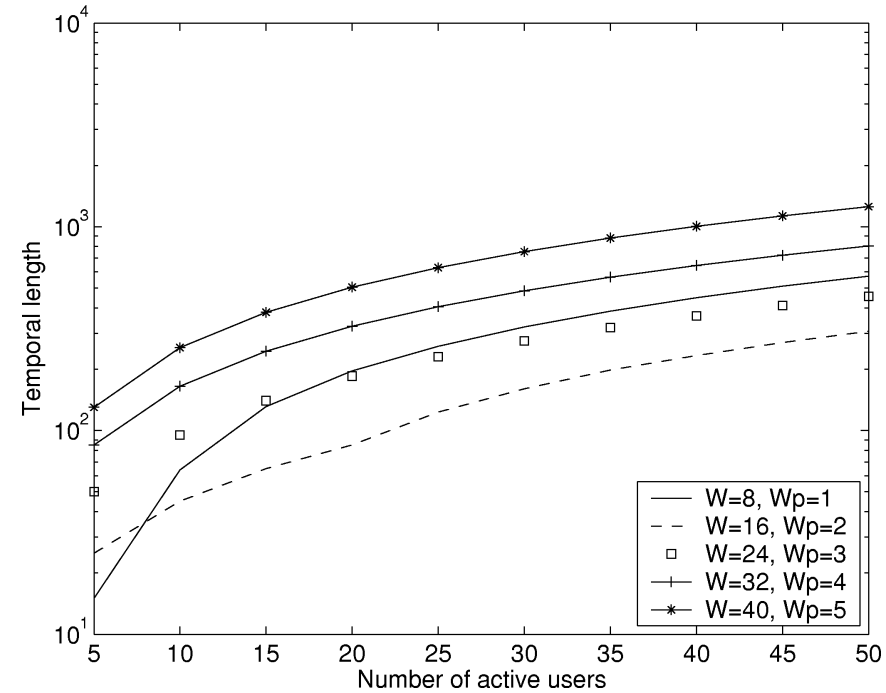

(a)

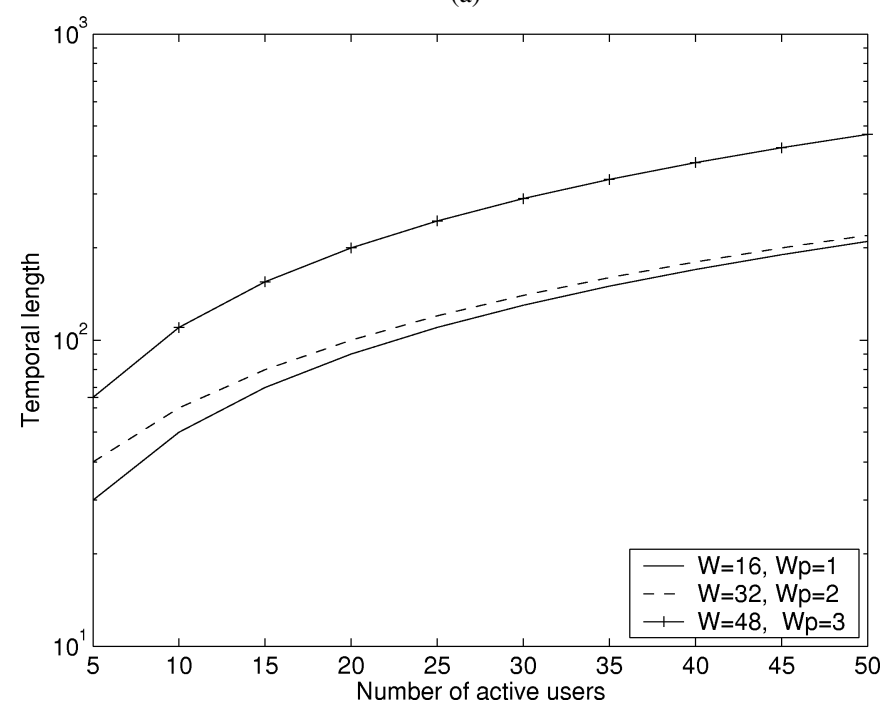

(b)

Fig. 6. Temporal lengths to achieve $P_{e}=10^{-9}$ when (a) $R=8$ and (b) $R=16$.

of the newly proposed codes were verified by simulation using Matlab. The performances of the W/T MPR codes and their limiting cases, W/T SPR and OOCs, are analyzed by considering only MAI, which is the major source of the noise in broadcast FO-CDMA networks. When the weight of the code is equal to 16 , the performance of the FO-CDMA networks using W/T MPR codes of $W_{p}=2$ is superior to the networks using other codes. In the limiting cases, W/T MPR codes approach OOCs and AML codes when the number of rows are equal to one and equal to the weight of the code, respectively. This paper compared various W/T codes and showed that W/T MPR codes have better cardinality and spectral efficiency for given wavelength $\times$ time dimensions, in addition to having minimal correlation values, which makes it suitable for FO-CDMA networks.

\section{ACKNOWLEDGMENT}

The authors would like to thank the anonymous reviewers for their useful suggestions and comments in improving the quality 
of this paper. The authors also thank Mr. P. Pattnaik and Mr. Madan for useful discussions.

\section{REFERENCES}

[1] J. A. Salehi, "Code division multiple access techniques in optical fiber networks part I: Fundamental principles," IEEE Trans. Commun., vol. 37, no. 8, pp. 824-833, Aug. 1989.

[2] L. Bin, "One-coincidence sequences with specified distance between adjacent symbols of frequency-hopping multiple access," IEEE Trans. Commun., vol. 45, no. 4, pp. 408-410, Apr. 1997.

[3] H. Fathallah, L. A. Rusch, and S. LaRochelle, "Passive optical fast frequency-hop CDMA communications systems," J. Lightw. Technol., vol. 17, no. 3, pp. 397-405, Mar. 1999.

[4] E. D. J. Smith, P. T. Gough, and D. P. Taylor, "Noise limits of optical spectral-encoding CDMA systems," Electron. Lett., vol. 31, no. 17, pp. 1469-1470, Aug. 17, 1995.

[5] E. D. J. Smith, R. J. Blaikie, and D. P. Taylor, "Performance enhancement of spectral-amplitude-encoding optical CDMA using pulse-position modulation," IEEE Trans. Commun., vol. 46, no. 9, pp. 1176-1185, Sep. 1998.

[6] P. K. Pepeljugoski, B. K. Whitlock, D. M. Kuchta, J. D. Crow, and S.-M. King, "Modeling and simulation of the OETC optical bus," in Conf. Proc. IEEE/LEOS'95 Meeting, vol. 1, 1995, pp. 185-186.

[7] B. K. Whitlock, P. K. Pepeljugoski, D. M. Kuchta, J. D. Crow, and S.-M. KIng, "Computer modeling and simulation of the optoelectronic technology consortium (OETC) optical bus," IEEE J. Sel. Areas Commun., vol. 15, no. 4, pp. 717-730, May 1997.

[8] L. Tancevski and I. Andonovic, "Wavelength hopping/time spreading code division multiple access systems," Electron. Lett., vol. 30, no. 17, pp. 1388-1390, Aug. 1994.

[9] - "Hybrid wavelength hopping/time spreading schemes for use in massive optical networks with increased security," J. Lightw. Technol., vol. 14 , no. 12, pp. 2636-2647, Dec. 1996.

[10] L. Tancevski, I. Andonovic, M. Tur, and J. Budin, "Massive optical LAN's using wavelength hopping/time spreading with increased security," IEEE Photon. Technol. Lett., vol. 8, no. 7, pp. 935-937, Jul. 1996.

[11] A. I. Mendez, R. M. Gagliardi, H. X. C. Feng, J. P. Heritage, and J.-M. Morookian, "Strategies for realizing optical CDMA for dense, high speed, long span, optical network applications," J. Lightw. Technol. (Special Issue on Optical Networks), vol. 18, no. 12, pp. 1685-1696, Dec. 2000.

[12] A. J. Mendez, V. J. Hernandez, C. V. Bennett, W. J. Lennon, and R. M. Gagliardi, "Optical CDMA (O-CDMA) technology demonstrator (TD) for 2D codes," in Proc. IEEE/LEOS'03 Meeting, pp. 1044-45. Paper ThEE3.

[13] A. J. Mendez, R. M. Gagliardi, V. J. Hernandez, C. V. Bennett, and W. J. Lennon, "Design and performance analysis of wavelength/time (W/T) matrix codes for optical CDMA," J. Lightw. Technol. (Special Issue on Optical Networks), vol. 21, no. 11, pp. 2524-2533, Nov. 2003.

[14] G. C. Yang and W. C. K. Wong, "Two-dimensional spatial signature patterns," IEEE Trans. Commun., vol. 44, no. 2, pp. 184-191, Feb. 1996.

[15] R. M. H. Yim, L. R. Chen, and J. Bajcsy, "Design and performance of 2D codes for wavelength-time optical CDMA," IEEE Photon. Technol. Lett., vol. 14, no. 5, pp. 714-716, May 2002.

[16] P. Patel, V. Baby, L. Xu, D. Rand, I. Glesk, and P. R. Prucna1, "A scalable wavelength-hopping, time-spreading optical CDMA system," in Proc. IEEE/LEOS'03 Meeting, Oct. 2003, pp. 1048-1049.
[17] E. S. Shivaleela, K. N. Sivarajan, and A. Selvarajan, "Design of a new family of two-dimensional codes for fiber-optic CDMA networks," $J$. Lightw. Technol., vol. 16, no. 4, pp. 501-508, Apr. 1998.

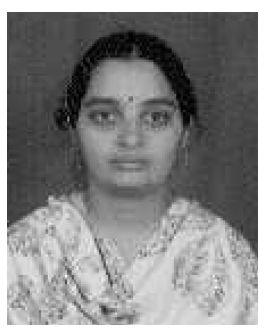

E. S. Shivaleela received the B.E. degree in electronics and communication from the Government BDT College of Engineering, Davanagere, Mysore University. She received the M.Sc.(Eng.) degree in electrical communication engineering from the Indian Institute of Science, Bangalore, India. She is currently working toward the Ph.D. degree ni the Department of Electrical Communication Engineering, Indian Institute of Science.

She currently works as Senior Scientific Officer with the Department of Electrical Communication Engineering, Indian Institute of Science. Her interests are mainly in the areas of all-optical networks and fiber and integrated optic components for photonic gigabit access systems.

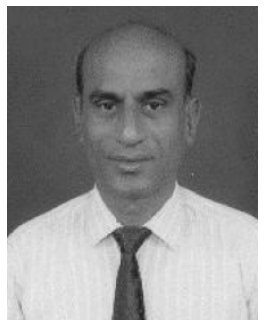

A. Selvarajan (M'01) received the M.Sc. degree in physics from Annamalai University, Tamil Nadu, India, and the Ph.D. degree from the Indian Institute of Science, Bangalore, India.

$\mathrm{He}$ is currently an Emiritus Professor with the Department of Electrical Communication Engineering, Indian Institute of Science. He has more than 150 publications in the areas of optics and spectroscopy, holography, acoustooptics, fiber optics, integrated optics, and optical communications. He has been a Visiting Scientist at the Optical Sciences Center, University of Arizona, Tempe; University of Upsala, Sweden; the Technical University of Denmark, Lyngby; University College London, London, U.K.; and the International Centre for Theoretical Physics, Trieste, Italy.

Dr. Selvarajan is a Member of the International Society for Optical Engineers (SPIE), the Optical Society of India, and the Institution of Electronics and Telecommunication Engineers (JETE).

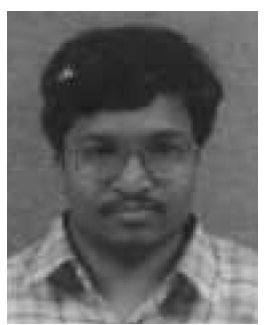

T. Srinivas (S'87-M'87) received the B.Sc. (Hon.) degree from Nehru Science College, Hydrabad, and M.E.(Int.) and Ph.D. degrees from the Indian Institute of Science, Bangalore, India.

He was a Postdoctoral Research Fellow at Toyohashi University of Technology, Japan, from 1992 to 1996. He is currently an Assistant Professor with the Department of Electrical Communication Engineering, Indian Institute of Science. His areas of interests are optical communication networks, integrated optics, micro-opto-electrical-mechanical systems (MOEMS), and fiber-optic sensors. 